\title{
Compensation effects in GaN:Mg probed by Raman spectroscopy and photoluminescence measurements
}

Cite as: J. Appl. Phys. 113, 103504 (2013); https://doi.org/10.1063/1.4794094

Submitted: 26 October 2012 . Accepted: 18 February 2013. Published Online: 08 March 2013

Ronny Kirste, Marc P. Hoffmann, James Tweedie, Zachary Bryan, Gordon Callsen, Thomas Kure, Christian Nenstiel, Markus R. Wagner, Ramón Collazo, Axel Hoffmann, and Zlatko Sitar

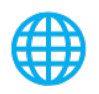

\section{ARTICLES YOU MAY BE INTERESTED IN}

\section{Luminescence properties of defects in $\mathrm{GaN}$}

Journal of Applied Physics 97, 061301 (2005); https://doi.org/10.1063/1.1868059

Heavy doping effects in Mg-doped GaN

Journal of Applied Physics 87, 1832 (2000); https://doi.org/10.1063/1.372098

First-principles calculations for defects and impurities: Applications to III-nitrides

Journal of Applied Physics 95, 3851 (2004); https://doi.org/10.1063/1.1682673

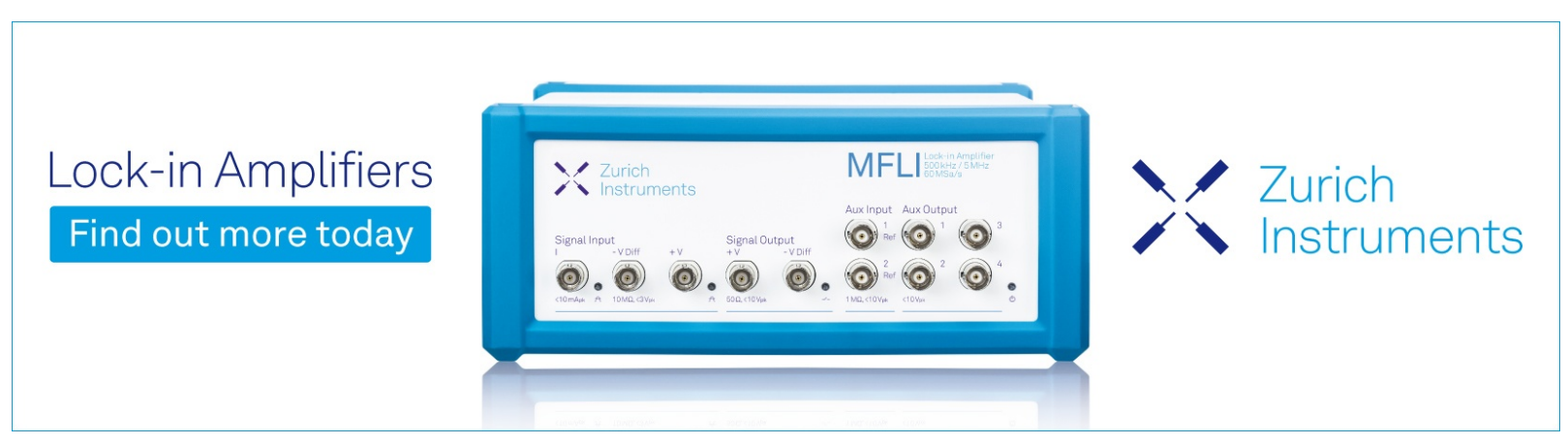




\title{
Compensation effects in GaN:Mg probed by Raman spectroscopy and photoluminescence measurements
}

\author{
Ronny Kirste, ${ }^{1,2, a)}$ Marc P. Hoffmann, ${ }^{2}$ James Tweedie, ${ }^{2}$ Zachary Bryan, ${ }^{2}$ Gordon Callsen, ${ }_{1}^{1}$ \\ Thomas Kure, ${ }^{1}$ Christian Nenstiel, ${ }^{1}$ Markus R. Wagner, ${ }^{1}$ Ramón Collazo, ${ }^{2}$ Axel Hoffmann, ${ }^{1}$ \\ and Zlatko Sitar ${ }^{2}$ \\ ${ }^{1}$ Institut für Festkörperphysik, TU Berlin, Hardenbergstraße 36, 10623 Berlin, Germany \\ ${ }^{2}$ Department of Material Science and Engineering, NCSU, 1001 Capability Dr., Raleigh, \\ North Carolina 27695, USA
}

(Received 26 October 2012; accepted 18 February 2013; published online 8 March 2013)

\begin{abstract}
Compensation effects in metal organic chemical vapour deposition grown GaN doped with magnesium are investigated with Raman spectroscopy and photoluminescence measurements. Examining the strain sensitive $\mathrm{E}_{2}(\mathrm{high})$ mode, an increasing compressive strain is revealed for samples with $\mathrm{Mg}$-concentrations lower than $7 \times 10^{18} \mathrm{~cm}^{-3}$. For higher $\mathrm{Mg}$-concentrations, this strain is monotonically reduced. This relaxation is accompanied by a sudden decrease in crystal quality. Luminescence measurements reveal a well defined near band edge luminescence with free, donor bound, and acceptor bound excitons as well as a characteristic donor acceptor pair (DAP) luminescence. Following recent results, three acceptor bound excitons and donor acceptor pairs are identified. Along with the change of the strain, a strong modification in the luminescence of the dominating acceptor bound exciton and DAP luminescence is observed. The results from Raman spectroscopy and luminescence measurements are interpreted as fingerprints of compensation effects in $\mathrm{GaN}: \mathrm{Mg}$ leading to the conclusion that compensation due to defect incorporation triggered by $\mathrm{Mg}$-doping already affects the crystal properties at doping levels of around $7 \times 10^{18} \mathrm{~cm}^{-3}$. Thereby, the generation of nitrogen vacancies is introduced as the driving force for the change of the strain state and the near band edge luminescence. (c) 2013 American Institute of Physics. [http://dx.doi.org/10.1063/1.4794094]
\end{abstract}

\section{INTRODUCTION}

Magnesium is the only useful p-dopant known so far for $\mathrm{GaN}$ and thus of high importance for nearly every (opto-) electronic application of $\mathrm{GaN}$ and its ternary compounds. The successful doping and activation of $\mathrm{Mg}$ were demonstrated in the early 1990s and widely investigated with numerous experimental techniques. ${ }^{1,2}$ Unfortunately, the maximum amount of free holes in $\mathrm{GaN}: \mathrm{Mg}$ is, despite intensive research, still limited to the $10^{17} \mathrm{~cm}^{-3}$ range, for which $\mathrm{Mg}$-concentrations in the range of $2 \times 10^{19} \mathrm{~cm}^{-3}$ are needed. $^{3}$ For higher $\mathrm{Mg}$ concentrations, the carrier concentration decreases, which is typically explained either by incorporation of $\mathrm{Mg}$ on interstitial sites, increased nitrogen vacancy concentration, and/or formation of inversion domains accompanied by the incorporation of oxygen. ${ }^{4}$ Although this problem has been known for a long time and is a technologically important aspect in the growth of devices, little progress has been made within the last few years in the understanding and control of these compensating defects.

Raman spectroscopy and photoluminescence measurements have proven to be powerful tools for the investigation of the optical, structural, and electrical properties of GaN., Magnesium incorporated on the Ga-site is expected to lead to compressive strain due to its larger ionic radius. This is expressed in a shift of the $\mathrm{E}_{2}$ (high) mode in the Raman spectra

\footnotetext{
a) Author to whom correspondence should be addressed. Electronic mail: rkirste@ncsu.edu.
}

towards higher energies. ${ }^{7}$ Furthermore, magnesium and any compensators may lead to the appearance of forbidden Raman modes due to an increased disorder in the crystal. ${ }^{8}$ In the luminescence measurements, the fingerprint of magnesium is a donor acceptor pair (DAP) and accordingly phonon replicas around $3.25 \mathrm{eV}$ as well as several acceptor bound exciton lines $(\mathrm{ABX})$ around $3.47 \mathrm{eV} .{ }^{9}$ Recently, the bound exciton lines were investigated very thoroughly and two or three different acceptors could be identified. ${ }^{6,10}$

In this contribution, Raman spectroscopy and luminescence measurements are used to investigate the incorporation of compensators as well as the connection between defects, strain, and the occurrence of acceptor bound exciton lines in GaN:Mg. The results presented within this work lead to an extended understanding of compensation effects and may help to overcome current limitations in the p-doping of GaN.

\section{EXPERIMENTAL}

Ga-polar GaN was grown by metal organic chemical vapour deposition (MOCVD) in a cold wall shower head reactor. ${ }^{11}$ The polarity was controlled by a low temperature AlN buffer grown on sapphire. ${ }^{12}$ In order to decrease dislocation and defect density, first a $1.2 \mu \mathrm{m}$ thick GaN layer was grown on this substrate at a temperature of $1050{ }^{\circ} \mathrm{C}$, reactor pressure of $20 \mathrm{Torr}$, and a V/III ratio of 100 . Finally, a $700 \mathrm{~nm}$ thick $\mathrm{GaN}$ layer doped with $\mathrm{Mg}$ was grown under similar conditions, only changing the V/III ratio to 200, whereby $\mathrm{Cp}_{2} \mathrm{Mg}$ served as the Mg-source. Thermal 
annealing was performed in a $\mathrm{N}_{2}$ purged tube furnace for $2 \mathrm{~h}$ ensuring fully activated samples. Using different $\mathrm{Cp}_{2} \mathrm{Mg}$ flows, the $\mathrm{Mg}$-concentration was varied from $8 \times 10^{17}$ to $2 \times 10^{19} \mathrm{~cm}^{-3}$ as confirmed by SIMS measurements. In order to gain information about the compensation, micro Raman measurements and photoluminescence measurements were performed. Raman spectra were recorded at room temperature using a DILOR XY setup equipped with a triple grating Raman spectrometer; the excitation source was the $488 \mathrm{~nm}$ line of an argon ion laser. ${ }^{4}$ The absolute error for the Raman measurements lies in the order of $0.2 \mathrm{~cm}^{-1}$ while the relative error can be estimated to be below $0.1 \mathrm{~cm}^{-1}$. For luminescence measurements, samples were mounted in a bath cryostat filled with liquid helium. Samples were excited with the $325 \mathrm{~nm}$ line of a $\mathrm{HeCd}$ laser. The luminescence signal was dispersed using a $1 \mathrm{~m}$ additive double-monochromator (Spex 1701). The signal was detected with a bi-alkali detector. Power dependent PL measurements were undertaken with a $\mathrm{XeCl}$ pumped dye laser (dye: p-terphenyl) with $100 \mathrm{MHz}$ repetition rate $\left(\lambda_{\mathrm{exc}}=340 \mathrm{~nm}\right)$.

\section{RESULTS}

Raman spectra of an undoped and a highly Mg-doped samples measured in $\mathrm{z}(\mathrm{xx}) \underline{\mathrm{z}}$ configuration are displayed in Fig. 1. In this configuration, the occurrence of the $E_{2}$ (high) and $\mathrm{A}_{1}(\mathrm{LO})$ mode is expected. ${ }^{5}$ It is seen from Fig. 1 that both modes are well pronounced in undoped as well as doped $\left(1 \times 10^{19} \mathrm{~cm}^{-3}\right)$ samples. Except for slight shifts of the modes, no differences between the two spectra are observed. This finding is in contrast to earlier results from Eckey et al. who observed a strengthening of the symmetry forbidden $\mathrm{E}_{1}(\mathrm{TO})$ and $\mathrm{A}_{1}(\mathrm{TO})$ modes with increasing $\mathrm{Mg}$-concentrations. ${ }^{8}$ Typically, the occurrence of forbidden Raman modes is assigned to a decrease in crystal quality (grain boundaries, multi reflections within the layer, disorder, etc.). In the case of Mg-doped GaN layers, the observation of the TO-modes was explained as an indicator of an increased defect incorporation due to the increased $\mathrm{Mg}$-doping and the $\mathrm{E}_{1}(\mathrm{TO}) /$ $\mathrm{E}_{2}$ (high) ratio was defined as a measure of the disorder in the sample. ${ }^{8}$ Following this argumentation, it could be concluded that for the investigated samples a comparable low defect incorporation or distortion of the crystal is apparent in all GaN layers, independent of the Mg-concentration. In addition to the GaN related Raman peaks, sometimes peaks which are identified as local vibrational modes from $\mathrm{Mg}$ are observed at energies from 640 to $675 \mathrm{~cm}^{-1} \cdot 13,14$ Thereby, the exact position of these peaks varies over a wide range. While none of these modes were observed in the presented samples, it should be mentioned that for some highly compressively strained samples, grown with different buffer layers (high temperature GaN instead of AlN), a strong peak around $650 \mathrm{~cm}^{-1}$ was observed (not shown). Furthermore, similar modes were observed in undoped and silicon doped samples as well. ${ }^{15}$ Therefore, an assignment of modes in this region to defect induced modes, similar as suggested by Kunert et al., seems to be very likely. ${ }^{13}$

The $\mathrm{E}_{2}$ (high) mode is non-polar meaning that its position is mainly influenced by strain and can be used as a

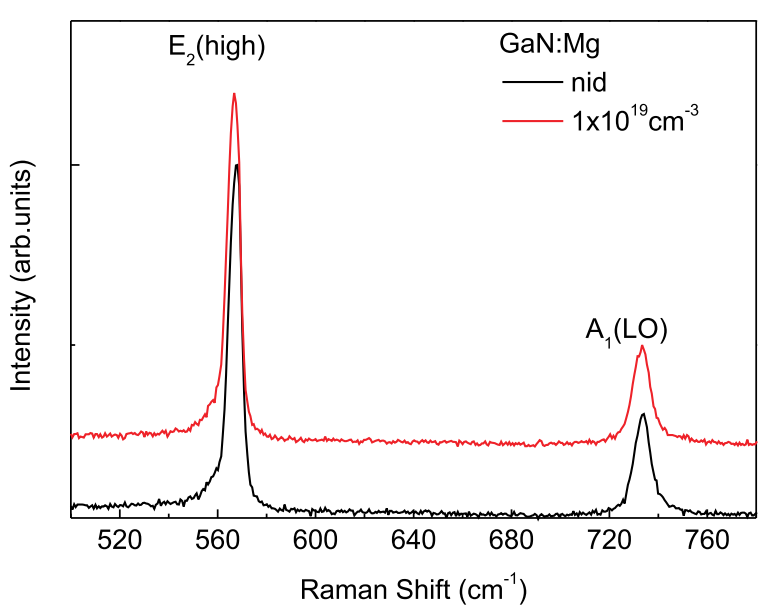

FIG. 1. Normalized room temperature Raman spectra of an undoped and a Mg-doped GaN samples.

metric for the strain state of a layer. Furthermore, the full width at half maximum (FWHM) is an indicator for defect incorporation in the layer since strain gradients or phonondefect scattering may lead to a broadening of the peak. The position and FWHM of the $\mathrm{E}_{2}$ (high) mode as a function of the Mg-concentration are displayed in Fig. 2. In accordance with the literature, ${ }^{16-18} 567 \mathrm{~cm}^{-1}$ is estimated for the relaxed position of the $\mathrm{E}_{2}$ (high) mode. For the undoped samples, a slight compressive strain is observed, which is related to the thermal and lattice mismatch between the GaN layer and sapphire substrate. ${ }^{16,17}$ For samples with low Mg concentration $\left(<4 \times 10^{18} \mathrm{~cm}^{-3}\right)$, a small increase in strain upon doping is observed in accordance with earlier results. ${ }^{8}$ However, for samples with higher $\mathrm{Mg}$ concentration $\left(>1 \times 10^{19} \mathrm{~cm}^{-3}\right)$, the $\mathrm{E}_{2}$ (high) mode shifts to lower energies, indicating a tensile strain. Simultaneously, the FWHM of the $E_{2}$ (high) undergoes a drastic change. Initially, the FWHM decreases slightly with increasing $\mathrm{Mg}$-doping, while for higher $\mathrm{Mg}$ concentrations a sudden increase of the FWHM is observed up to a level well above that of undoped GaN. This indicates a change in the defect incorporation for samples doped with more than $7 \times 10^{18} \mathrm{~cm}^{-3} \mathrm{Mg}$. It is suggested that this change is triggering the change of the strain state from compressive to tensile.

In contrast to the $\mathrm{E}_{2}$ (high) mode, the $\mathrm{A}_{1}(\mathrm{LO})$ mode is polar. Thus, a shift and broadening of this mode is expected for increasing carrier concentration due to phonon-plasmon coupling (LOPC). ${ }^{19,20}$ However, such a shift or broadening was not observed in the investigated samples, indicating a strong plasmon damping as it was observed in Mg-doped $\mathrm{GaN}$ before. $^{8,21}$

Luminescence measurements have proven to be a versatile tool to investigate $\mathrm{Mg}$-doping and compensation. ${ }^{22}$ Recently, detailed studies revealed that luminescence spectra of Mg-doped GaN may contain two or three ABX and associated DAP transitions. ${ }^{6,10}$ The low temperature luminescence signal in the range of the DAP and ABX is shown in Fig. 3. For the undoped sample, the near band edge signal consists of a $\mathrm{Si}$ or $\mathrm{O}$ bound exciton accompanied by weaker free $\mathrm{A}$ and $\mathrm{B}$ excitons. ${ }^{23}$ No DAP luminescence is observed 


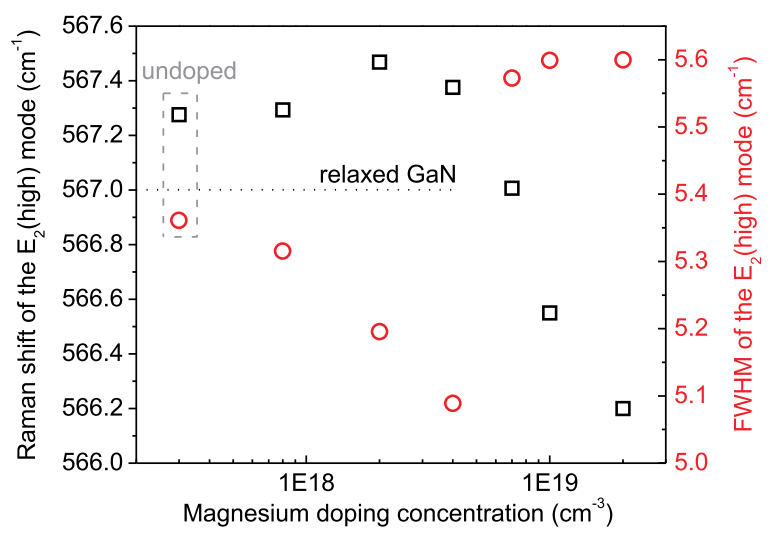

FIG. 2. Position (squares) and FWHM (circles) of the strain sensitive $\mathrm{E}_{2}$ (high) Raman mode depending on the Mg-concentration. Additionally, position and FWHM of an undoped sample are shown (dashed box).

for this sample. ${ }^{22}$ For lowly doped GaN:Mg samples, a strong ABX1 emission dominates the spectrum. This ABX1 was recently assigned to a neutral acceptor bound exciton. ${ }^{6}$ The corresponding DAP emission for lowly doped samples is the DAP1, which is the recombination from the $\mathrm{Si} / \mathrm{O} \mathrm{DBX}$ and the $\mathrm{ABX} 1$. For higher Mg-concentrations, the dominating emissions strongly change. For the near band edge luminescence, the ABX3 becomes the dominating emission, while in the DAP region the DAP3 rises. It should be noted that the energetic split between DAP1 and DAP3 is $29 \mathrm{meV}$, which exactly matches the split between the ABX1 and ABX3 transition. The transition from $\mathrm{ABX} 1$ to $\mathrm{ABX} 3$ and DAP1 to DAP3 occurs at $\mathrm{Mg}$-concentrations around $4 \times 10^{18} \mathrm{~cm}^{-3}$ to $7 \times 10^{18} \mathrm{~cm}^{-3}$. At these concentrations, the luminescence spectra are a mixture between DAP1 and DAP3. In addition to the $\mathrm{ABX} 1$ and $\mathrm{ABX} 3$, a peak identified as $\mathrm{ABX} 2$ can be observed at energies around $3.458 \mathrm{eV}$. This peak is observable in all doped samples but is typically observed with intensity below that of the ABX1 or ABX3.

For the deeper defect related emission, only the yellow luminescence is observed (not shown). ${ }^{23}$ This yellow luminescence is at a comparably low level in all samples. Typically, this yellow luminescence is identified as either carbon or Gallium-vacancy related, whereby newer theoretical calculation point towards carbon as the origin. ${ }^{24}$ Taking into account that all samples were grown at the same supersaturation rate, which implies comparable carbon incorporation in all samples, this finding is a strong indicator that carbon incorporation is not influenced by $\mathrm{Mg}$-doping (thus, it does not act as a compensator). ${ }^{11}$

Power dependent PL measurements of the luminescence of the GaN:Mg sample with $2 \times 10^{18} \mathrm{~cm}^{-3}$ magnesium concentration are shown in Fig. 4. For low excitation densities, as achieved with a HeCd laser (Fig. 4-bottom most spectrum), the spectrum consists of an ABX3 and a DAP3 including its phonon replicas. If the excitation source is switched to the $\mathrm{XeCl}$ pumped dye laser (pulsed at $100 \mathrm{~Hz}$ ), the excitation density drastically increases leading to the occurrence of peaks at higher energies, namely, the DAP1/2 and the $\mathrm{ABX} 2$. A further increase in the excitation density leads to a saturation of the DAP luminescence and a strong, dominant ABX signal.

\section{DISCUSSION}

Magnesium, if incorporated on Ga-site, is expected to lead to a compressive strain due to its larger ionic radius as compared to Ga. Thus, an increasing compressive strain for higher $\mathrm{Mg}$-concentrations is expected. ${ }^{8}$ However, if any other defects are incorporated in parallel, these defects might lead to an additional tensile or compressive strain which overlays the Mg-induced strain. For the investigated samples, the $\mathrm{E}_{2}$ (high) mode shifts towards higher energies upon $\mathrm{Mg}$-doping, which suits to the incorporation of $\mathrm{Mg}$ on Ga-site. For Mg-concentrations above $7 \times 10^{18} \mathrm{~cm}^{-3}$, the strain decreases and finally gets slightly tensile. This is a strong indicator that an additional defect is incorporated with at least similar concentration, which balances the compressive strain. This finding is supported by the FWHM of the $\mathrm{E}_{2}$ (high) mode, which suddenly increases at these $\mathrm{Mg}$ -

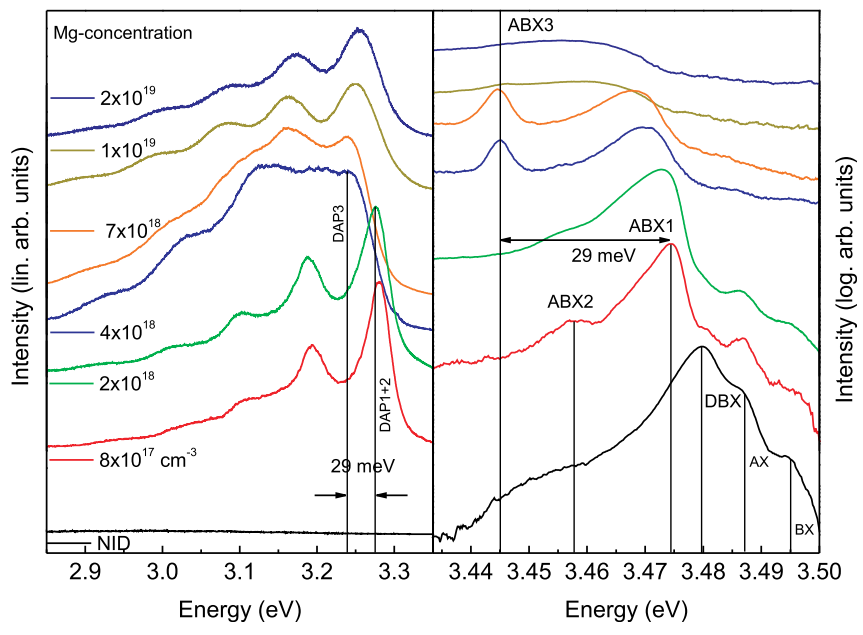

FIG. 3. Low temperature $(2 \mathrm{~K})$ luminescence signal in the region of the donor acceptor pair transition (left) and the near band edge luminescence (right) from samples with different $\mathrm{Mg}$-concentrations.

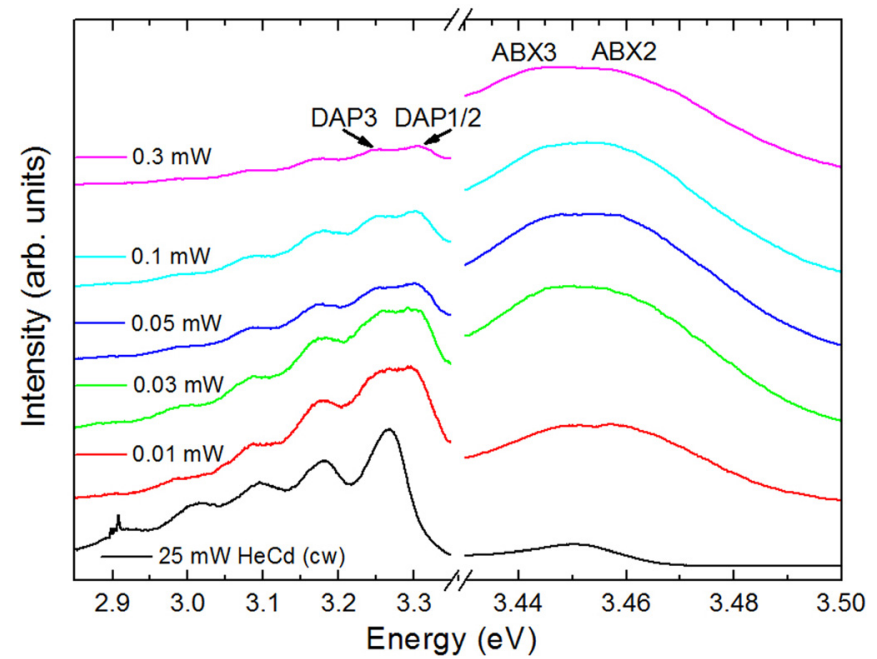

FIG. 4. Power dependent low temperature PL spectra of a GaN:Mg sample with $2 \times 10^{19} \mathrm{~cm}^{-3}$ magnesium concentration. 
concentrations. For increasing Mg-concentrations, the Fermi level shifts towards the valence band maximum (VBM) favoring the incorporation of compensating defects by lowering their formation energy. ${ }^{25,26}$ It has been found that especially the formation energies of hydrogen and nitrogen vacancies are strongly reduced, as the Fermi energy approaches the VBM. Hydrogen is not expected to be in the samples with amounts above the background concentration, since the samples were annealed and fully activated. Furthermore, hydrogen would be incorporated as $\mathrm{Mg}-\mathrm{H}$ complex leading to a compressive strain. In addition, carbon, another common defect in $\mathrm{GaN}$ was found to be incorporated at similar amounts, as proven by the intensity of the yellow luminescence. Nitrogen vacancies, on the other hand, would lead to a tensile strain and are known to be strong electrical compensators for Mg-concentrations above $2 \times 10^{19} \mathrm{~cm}^{-3} \cdot{ }^{27}$ Thus, the decreasing strain (and later tensile strain) in the investigated samples is identified as the fingerprint of the formation of nitrogen vacancies, indicating that the properties of $\mathrm{GaN}: \mathrm{Mg}$ are already influenced by this defect type at $\mathrm{Mg}$ concentrations as low as $7 \times 10^{18} \mathrm{~cm}^{-3}$. The increasing compressive strain for higher $\mathrm{Mg}$ concentration as observed in earlier works might be explained by not fully activated samples or the incorporation of other defects such as inversion domains or oxygen. ${ }^{8}$

In addition to the change of the strain state, a change of the optical spectra is observed for $\mathrm{Mg}$-concentrations above $7 \times 10^{18} \mathrm{~cm}^{-3}$. While for lower concentration, the ABX1 and DAP1 (with some contributions from ABX2) are dominating the spectra, at higher Mg-concentrations, the $\mathrm{ABX} 3$ and DAP3 are dominating the spectra. This change in the spectral shape of the samples is attributed to the different defect incorporation upon increasing $\mathrm{Mg}$-concentration. In contrast to the work of Monemar et al., an assignment of the $\mathrm{ABX} 1$ to a Mg-H complex can be excluded since all samples were fully activated and the spectra did not change with longer laser excitation time. ${ }^{10}$ However, Callsen et al. were pointing out that $A B X 1$ is a shallow acceptor, while $A B X 3$ is related to a deep acceptor. ${ }^{6}$ It is obvious that the change in the strain state and the change in the optical spectra occurs simultaneously, indicating some relation between the increased nitrogen vacancy incorporation and the $\mathrm{ABX} 3$ transition, perhaps by the formation of complexes. ${ }^{28}$

Interestingly, the $\mathrm{ABX} 2$ luminescence is observable in all samples. For low Mg-concentrations, the ABX2 can be observed as a single peak $\left(8 \times 10^{17} \mathrm{~cm}^{-3}\right.$ Fig. 3). With an increasing $\mathrm{Mg}$ concentration, this peak is only observable as a part of the flank of the ABX1 and later on the ABX3 transition. However, power dependent measurements (Fig. 4) demonstrated that the intensity of the $\mathrm{ABX} 3$ increases with respect to the ABX1 for higher excitation densities. Similar observations are made for the related DAP2 transition. The increasing intensity is related to the filling of low energy levels at higher excitation power densities. This finding indicates that the acceptor related to the ABX2/DAP2 is incorporated at a comparable level in all samples and is independent of the $\mathrm{Mg}$-concentration. In contrast, no rise in the ABX1/DAP1 transition is observed, indicating the absence of these acceptors states in the samples.

\section{CONCLUSION}

In conclusion, it is demonstrated how the incorporation of defects (compensators) can influence and control the strain and optical properties of GaN:Mg. Therefore, the influence of $\mathrm{Mg}$-doping on the vibrational and optical properties of $\mathrm{GaN}$ was investigated with Raman spectroscopy and photoluminescence, respectively. It was found that GaN with $\mathrm{Mg}$-concentrations up to $2 \times 10^{19} \mathrm{~cm}^{-3}$ has a high crystal quality as indicated by the absence of any symmetry forbidden modes and comparable narrow FWHM of the Raman modes. A change of the strain state from compressive to tensile is explained by the generation of nitrogen vacancies. This change of the strain state is accompanied by a transition from a shallow to deep acceptor state.

\section{ACKNOWLEDGMENTS}

This work was supported by the DFG within SFB787 and the NSF within Grant No. 1108071.

${ }^{1}$ H. Amano, M. Kito, K. Hiramatsu, and I. Akasaki, Jpn. J. Appl. Phys. 28(12), L2112 (1989).

${ }^{2}$ S. Nakamura, T. Mukai, M. Senoh, and N. Iwasa, Jpn. J. Appl. Phys. (31)(2B), L139-L142 (1992).

${ }^{3}$ H. Obloh, K. H. Bachem, U. Kaufmann, M. Kunzer, M. Maier, A. Ramakrishnan, and P. Schlotter, J. Cryst. Growth 195(1-4), 270-273 (1998).

${ }^{4}$ R. Kirste, R. Collazo, G. Callsen, M. R. Wagner, T. Kure, J. S. Reparaz, S. Mita, J. Xie, A. Rice, J. Tweedie, Z. Sitar, and A. Hoffmann, J. Appl. Phys. 110(9), 093503-093509 (2011).

${ }^{5}$ M. Kuball, Surf. Interface Anal. 31(10), 987-999 (2001).

${ }^{6}$ G. Callsen, M. R. Wagner, T. Kure, J. S. Reparaz, M. Bügler, J. Brunnmeier, C. Nenstiel, A. Hoffmann, M. Hoffmann, J. Tweedie, Z. Bryan, S. Aygun, R. Kirste, R. Collazo, and Z. Sitar, Phys. Rev. B 86(7), 075207 (2012).

${ }^{7}$ A. Kaschner, H. Siegle, A. Hoffmann, C. Thomsen, U. Birkle, S. Einfeldt, and D. Hommel, MRS Internet J. Nitride Semicond. Res. 4S1, G3.57 (1999).

${ }^{8}$ L. Eckey, U. Von Gfug, J. Holst, A. Hoffmann, B. Schineller, K. Heime, M. Heuken, O. Schön, and R. Beccard, J. Cryst. Growth 189-190, 523-527 (1998)

${ }^{9}$ A. K. Viswanath, E.-J. Shin, J. I. Lee, S. Yu, D. Kim, B. Kim, Y. Choi, and C.-H. Hong, J. Appl. Phys. 83, 2272 (1998).

${ }^{10}$ B. Monemar, P. P. Paskov, G. Pozina, C. Hemmingsson, J. P. Bergman, T. Kawashima, H. Amano, I. Akasaki, T. Paskova, S. Figge, D. Hommel, and A. Usui, Phys. Rev. Lett. 102(23), 235501 (2009).

${ }^{11}$ S. Mita, R. Collazo, A. Rice, R. F. Dalmau, and Z. Sitar, J. Appl. Phys. 104(1), 013521-013529 (2008).

${ }^{12}$ S. Mita, R. Collazo, and Z. Sitar, J. Cryst. Growth 311(10), 3044-3048 (2009).

${ }^{13}$ H. W. Kunert, D. J. Brink, F. D. Auret, M. Maremane, L. C. Prinsloo, J. Barnas, B. Beaumont, and P. Gibart, Mater. Sci. Eng., B 102(1-3), 293-297 (2003).

${ }^{14}$ H. Harima, T. Inoue, S. Nakashima, M. Ishida, and M. Taneya, Appl. Phys. Lett. 75(10), 1383-1385 (1999).

${ }^{15}$ S. Fritze, A. Dadgar, H. Witte, M. Bugler, A. Rohrbeck, J. Blasing, A. Hoffmann, and A. Krost, Appl. Phys. Lett. 100(12), 122104 (2012).

${ }^{16}$ L. Filippidis, H. Siegle, A. Hoffmann, C. Thomsen, K. Karch, and F. Bechstedt, Phys. Status Solidi B 198(2), 621-627 (1996).

${ }^{17}$ G. Callsen, J. S. Reparaz, M. R. Wagner, R. Kirste, C. Nenstiel, A. Hoffmann, and M. R. Phillips, Appl. Phys. Lett. 98(6), 061906 (2011).

${ }^{18}$ U. Haboeck, H. Siegle, A. Hoffmann, and C. Thomsen, Phys. Status Solidi C 0(6), 1710-1731 (2003).

${ }^{19}$ P. Perlin, J. Camassel, W. Knap, T. Taliercio, J. C. Chervin, T. Suski, I. Grzegory, and S. Porowski, Appl. Phys. Lett. 67(17), 2524-2526 (1995).

${ }^{20}$ R. Kirste, S. Mohn, M. R. Wagner, J. S. Reparaz, and A. Hoffmann, Appl. Phys. Lett. 101(4), 041904-041909 (2012). 
${ }^{21}$ G. Popovici, G. Y. Xu, A. Botchkarev, W. Kim, H. Tang, A. Salvador, H. Morkoc, R. Strange, and J. O. White, J. Appl. Phys. 82(8), 4020-4023 (1997).

${ }^{22}$ M. A. Reshchikov, G. C. Yi, and B. W. Wessels, Phys. Rev. B 59(20), 13176-13183 (1999).

${ }^{23}$ M. A. Reshchikov and H. Morkoc, J. Appl. Phys. 97(6), 061301-061395 (2005).

${ }^{24}$ J. L. Lyons, Appl. Phys. Lett. 97(15), 152108 (2010).
${ }^{25}$ J. Neugebauer and C. G. Van de Walle, Phys. Rev. B 50(11), 8067-8070 (1994).

${ }^{26}$ C. G. Van de Walle and J. Neugebauer, J. Appl. Phys. 95(8), 3851-3879 (2004).

${ }^{27}$ U. Kaufmann, P. Schlotter, H. Obloh, K. Köhler, and M. Maier, Phys. Rev. B 62(16), 10867-10872 (2000).

${ }^{28}$ C. D. Latham, R. Jones, S. Öberg, R. M. Nieminen, and P. R. Briddon, Phys. Rev. B 68(20), 205209 (2003). 